\title{
US Budget Impact Model for Selinexor in Relapsed or Refractory Multiple Myeloma
}

This article was published in the following Dove Press journal:

ClinicoEconomics and Outcomes Research

\begin{abstract}
Jan Bassali'
Ian Gopal Gould ${ }^{2}$

James A Kaye $\mathbb{D}^{3}$

Deirdre Mladsi (iD) ${ }^{4}$

Jyotsna Mehta $\mathbb{D I D}^{5}$
\end{abstract}

'Global Medical and Scientific Affairs Department , Karyopharm Therapeutics, Newton, MA, 02459, USA; ${ }^{2}$ Research Health Economy, RTI Health Solutions, Research Triangle Park, NC 27709-2194, USA; ${ }^{3}$ Epidemiology, RTI Health

Solutions, Research Triangle Park, NC, USA; ${ }^{4}$ Health Economics - Strategy and Early Modeling, RTI Health Solutions, Research Triangle Park, NC, USA; ${ }^{5}$ HEOR, Karyopharm Therapeutics, Newton, MA 02459, USA
Correspondence: Jan Bassali Karyopharm Therapeutics, Newton, MA 02459, USA

$\mathrm{Tel}+1617658-0600$

Email jbassali@karyopharm.com
Objective: To estimate the budgetary impact of adopting selinexor (XPOVIO; Karyopharm Therapeutics, Inc.) for the treatment of adult patients with penta-refractory multiple myeloma (MM) from the perspective of a third-party payer in the United States (US).

Methods: A budget impact analysis was conducted in one-year increments for the first 3 years after the introduction of selinexor for a private payer or Medicare Part D. Total annual treatment costs (2018 US dollars) were calculated as the sum of drug costs, costs of adverse events (AEs; grade $\geq 3$ ), along with ongoing best supportive care costs. The number of eligible patients was derived from national epidemiology statistics, healthcare databases, and published literature.

Results: In the base-case analysis, selinexor was associated with a per member per month (PMPM) cost of $\$ 0.0103$ in year 3, assuming a market uptake of $64 \%$, for a hypothetical private payer plan with one million members and four eligible patients. In a scenario analysis with 16 eligible patients with triple-class refractory MM regardless of the line of therapy (this additional scenario analysis was performed with an eligible population that does not fit squarely within the approved label for selinexor but was performed strictly for the purpose of demonstrating the results of the budget impact model when based on a larger pool of eligible patients), the estimated PMPM cost in year 3 was $\$ 0.0388$. The model showed comparable sensitivity to treatment duration, wholesale acquisition cost for selinexor, and year 1 uptake. The base-case analysis conducted from the perspective of Medicare Part D was associated with a PMPM cost of $\$ 0.0078$ in year 3 with 159 eligible patients.

Conclusions: The model estimates a small and manageable budget impact of adopting selinexor into a third-party US payer plan, given the low prevalence of penta-refractory MM.

Keywords: multiple myeloma, budget impact analysis, Selinexor, US

\section{Introduction}

Multiple myeloma (MM) is a hematologic cancer that develops as a plasma cell malignancy within the bone marrow. ${ }^{1}$ Clinical manifestations of $\mathrm{MM}$ are driven by the production and secretion of monoclonal proteins and other bioactive molecules by malignant cells and are associated with end organ damage, including hypercalcemia, renal insufficiency or failure, anemia, immune dysfunction, and bone destruction. MM is the second most common form of blood cancer in the US, with an estimated prevalence of 131,392 patients with MM in 2016. ${ }^{2}$ Projections for 2019 included 32,110 estimated new cases of MM and 12,960 estimated deaths from the disease in the US. ${ }^{3}$

Significant progress has been made in the treatment of MM over the past two decades with the development of novel therapeutics and immunotherapies. ${ }^{4,5}$ There 
are currently three classes of drugs used, often in combination, for the treatment of MM: proteasome inhibitors (PIs; eg, bortezomib, carfilzomib), immunomodulatory agents (IMIDs; eg, lenalidomide, pomalidomide), and monoclonal antibodies (eg, the anti-CD38 monoclonal antibody daratumumab, the anti-CS1 antibody elotuzumab). ${ }^{4,5}$ Additional treatment modalities include chemotherapy, bone marrow transplant, and radiation therapy. Newer therapies, including IMIDs and PIs, were utilized by $61.3 \%$ of patients in $2014 .^{6}$

Despite advances made in treatment, the disease is incurable and almost all patients experience relapse and develop refractory (drug-resistant) disease. The 5-year survival rate for patients diagnosed with MM from 2009 to 2015 was estimated to be $52.2 \%$ in the US. ${ }^{2}$ Treatment of relapsed and/or refractory MM (RRMM) is particularly challenging, with relatively poor prognosis for most patients. ${ }^{4,7,8}$ For patients with MM that is refractory to multiple therapies, overall survival (OS) is extremely short. For example, in heavily pretreated patients with daratumumab-refractory $\mathrm{MM}$, the median $\mathrm{OS}$ is 1.7 to 3.0 months. ${ }^{9-12}$ Having exhausted all available treatment options with clinical benefit, patients may choose between experimental therapy, retreatment strategies, and best supportive care for managing symptoms and quality of life. ${ }^{12,13}$

Selinexor (XPOVIO; Karyopharm Therapeutics, Inc.) is a first-in-class selective oral nuclear transport inhibitor that works synergistically with dexamethasone to achieve selective killing of malignant plasma cells. ${ }^{14}$ In the STORM (Selinexor Treatment of Refractory Myeloma) phase $2 \mathrm{~b}$ trial (NCT02336815), patients $(\mathrm{N}=122 ; \mathrm{n}=83$ with penta-refractory myeloma, ie, refractory to bortezomib, carfilzomib, lenalidomide, pomalidomide, and daratumumab) had an overall response rate (ORR) of $25 \%$ $(\mathrm{N}=21 ; 95 \%$ confidence interval [CI], 19 to 35), and a median OS of 8.6 months (95\% CI, 6.2 to 11.3$){ }^{15,16}$ Selinexor has been approved by the US Food and Drug Administration (FDA) in combination with low dose dexamethasone for the treatment of adult patients with RRMM who have received at least four prior therapies and whose disease is refractory to at least two PIs, two IMIDs, and an anti-CD38 monoclonal antibody. ${ }^{14}$

The introduction of newer drug therapies has been associated with an increase in the costs of RRMM treatment. According to a retrospective analysis of the Truven Health MarketScan Research Databases, the per patient per month (PPPM) MM treatment-related drug costs rose from $\$ 346$ in 2000 to $\$ 4,179$ in $2014 .^{6}$ In 2014 , treatment- related drug costs accounted for an estimated $28.5 \%$ of total healthcare costs among patients with $\mathrm{MM} .{ }^{6}$ The cost of oncology drugs has a significant impact on payers' budgets, and quantifying the potential budget impact of new therapies is important for the management of oncology costs. ${ }^{17}$ Value-based frameworks also incorporate economic evidence to inform healthcare decision-makers of the potential value of new therapies. ${ }^{18}$ The American Society of Clinical Oncology (ASCO), for example, utilizes cost of oncology drugs in the calculation of net health benefits. ${ }^{19,20}$ In this work, a US-focused model was developed to evaluate the expected budget impact of adopting selinexor into a third-party payer plan for the treatment of eligible adult patients with RRMM.

\section{Methods}

\section{Model Characteristics: Perspective, Time Horizon, Structure}

The net budgetary impact of selinexor was considered from the perspective of a third-party payer in the US. The budget impact analysis followed the International Society for Pharmacoeconomics and Outcomes Research (ISPOR) guidelines as well as the guidelines set forth by the Academy of Managed Care Pharmacy (AMCP) for economic evaluations to support formulary listings. ${ }^{21,22}$ A schematic of the budget impact model is shown in Figure 1.

The model considered only direct costs associated with selinexor treatment. Total annual treatment costs (ie, drug costs, costs of serious adverse events, and best supportive care costs) were calculated to determine the projected budget impact of adding selinexor as a treatment for eligible patients. The model did not include discounting. All results are presented undiscounted as is standard practice and aligned with AMCP requirements and ISPOR guidelines. ${ }^{21,23} \mathrm{~A}$ key assumption was that the use of selinexor would not meaningfully reduce the costs of best supportive care as patients would continue to receive intervention through supportive care to address complications of disease arising from anemia and bone disease or destruction (eg, bone pain). ${ }^{24}$ The analysis was conducted in one-year increments for the first 3 years after the introduction of selinexor.

\section{Model Population}

The model population was based on the projected population of the US in 2019 (US Census Bureau). ${ }^{25}$ The input for the annual number of US patients who were considered 


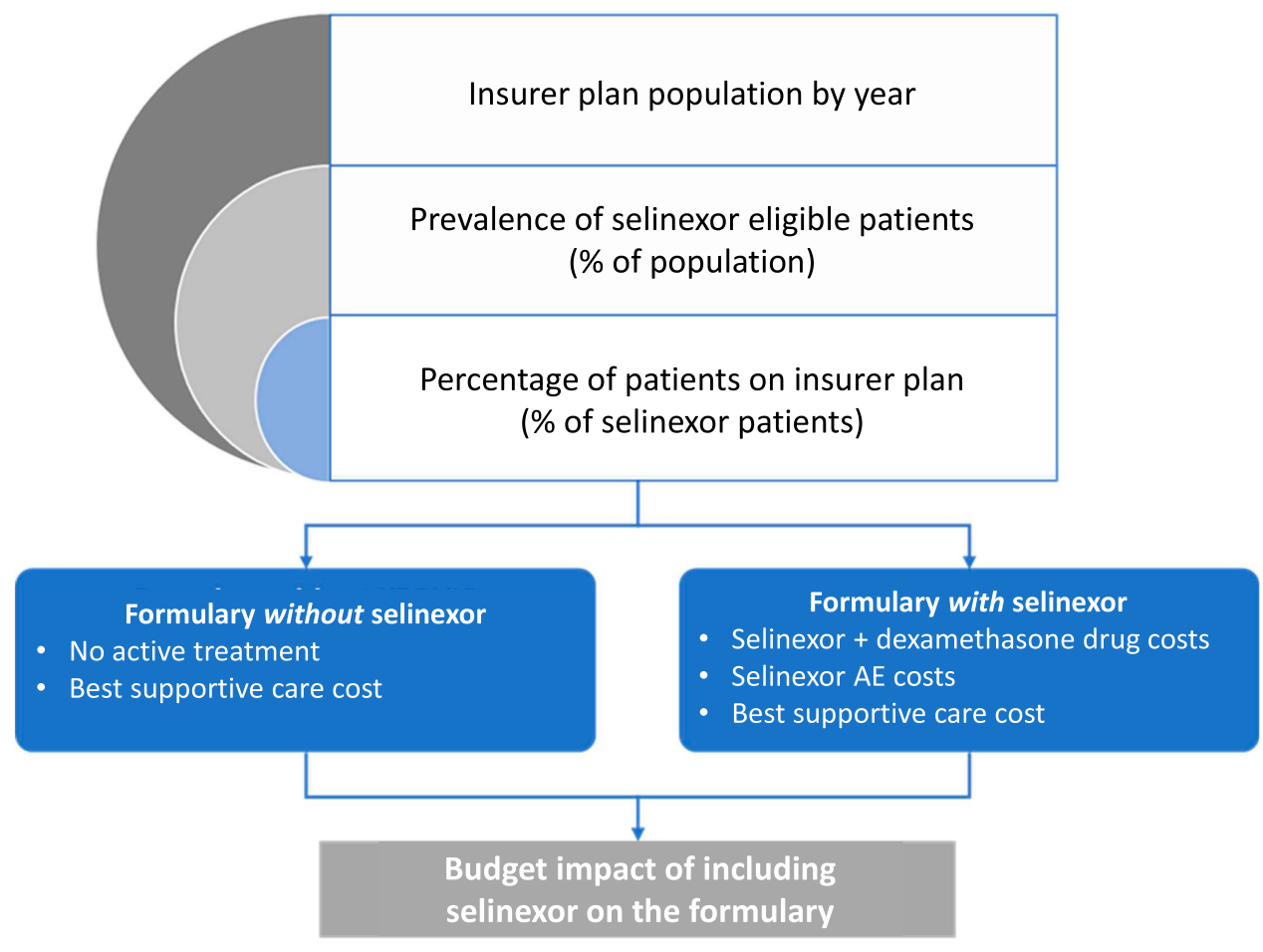

Figure I Schematic representation of the budget impact model structure.

eligible to receive selinexor was $2,400 .^{26,27}$ The selinexor eligible patient population size was derived from the estimated number of patients in therapy line 3 or greater $(4 \mathrm{~L}+)$ with prior bortezomib, lenalidomide, daratumumab, pomalidomide, and carfilzomib or ixazomib exposure. Sources used to estimate the eligible patient population size included the Surveillance, Epidemiology, and End Results (SEER) program, Kantar Health, Intrinsiq, Symphony Claims Data (2016 to 2018), and the Flatiron Health Analytic Database (2011 to 2018).

Based on epidemiology studies, approximately $60 \%$ of patients with RRMM are above the age of $65 .^{28-30}$ Because of differences by age in mortality rates and likelihood to proceed through multiple lines of treatment, younger patients may be more likely to be eligible for selinexor in the penta-refractory setting. Of patients eligible for treatment with selinexor, $57 \%$ were assumed to be on a private insurer plan (ie, younger than 65) while $43 \%$ were assumed to be on Medicare (ie, aged 65 or older). As such, two base-case perspectives were considered for the model; one from the perspective of a private insurer and a second from the perspective of Medicare.

As a scenario analysis, the model population could be modified to reflect the estimated number of US patients with triple-class refractory MM who have received three previous lines of therapy (ie, 6,000 patients) or the number of patients with triple-class refractory MM regardless of the number of prior lines of therapy (ie, 9,000 patients). ${ }^{26}$ This additional scenario analysis was performed with an eligible population that does not fit squarely within the approved label for selinexor but was performed strictly for the purpose of demonstrating the results of the budget impact model when based on a larger pool of eligible patients.

The model considered a hypothetical private payer plan with population of one million members, or, alternatively, 51 million members participating in Medicare Part D. ${ }^{31,32}$ An assumption was made that the number of selinexor eligible patients would remain constant over 3 years.

\section{Treatment Parameters Medication Dosing, Duration, and Treatment Costs}

The budget impact model included selinexor therapy as provided in the STORM trial (NCT02336815) and best supportive care. ${ }^{33}$ The model used a dosage of $80 \mathrm{mg}$ twice weekly for selinexor and a concurrent dosage of $20 \mathrm{mg}$ for oral dexamethasone. The unit cost for dexamethasone was set to the wholesale acquisition cost (WAC) obtained from Red Book Online (Micromedex 2.0 2018). ${ }^{34}$ The WAC of selinexor was based on data on file (Karyopharm Inc.). ${ }^{35}$ The WAC per package of selinexor 
containing 32 tablets of $20 \mathrm{mg}$ strength was $\$ 22,000$. All costs in the model were presented in 2018 US dollars. In the base-case analysis, no WAC discount was assumed.

The median duration of treatment with selinexor and concurrent dexamethasone was 8 weeks according to the prescribing information for selinexor. ${ }^{14}$ The model assumed that every patient received selinexor and dexamethasone for a total of 8 weeks in 52 weeks (ie, 1 year), along with continuous best supportive care for 52 weeks in 1 year. Default inputs for calculation of selinexor treatment costs are summarized in Table 1. The average per person drug cost for 1 year of treatment was calculated by accounting for the cost of drugs (ie, WAC), dosage, frequency, and duration of treatment. As both selinexor and dexamethasone are orally dosed, no wastage was considered in the model. In the basecase analysis, adherence-adjustment factors (ie, drug adherence) for selinexor and dexamethasone were set to $100 \%$. It was assumed that drug costs were not reduced by costsharing through co-insurance or co-payment. ${ }^{36}$

The cost of best supportive care was estimated based on a previous study that examined costs of managing bone pain and anemia in patients with MM receiving their third line of therapy. ${ }^{24}$ The reported PMPM cost of $\$ 567$ was multiplied by 12 to determine the per patient annual cost and inflated to 2018 costs using the personal consumption expenditure (PCE) inflation index. ${ }^{37}$ Thus, the input for per patient annual cost of best supportive care was $\$ 6,893$.

\section{Market Share}

The model assumed a year 1 uptake of $28 \%$. The market penetration was assumed to increase to $64 \%$ in year 2 . A peak market share of $64 \%$ was maintained at year $3 .^{38}$ These estimates are based on interviews conducted with US physicians who treat patients with RRMM, treatment patterns for highly pretreated RRMM in the US, and market projections.

\section{Adverse Events}

The model considered grade 3 or higher treatment-emergent adverse events (TEAEs) from selinexor occurring in at least $5 \%$ of patients as reported in the STORM Clinical Study Report. $^{33,39}$ The model made a conservative assumption that grade 3 and higher TEAEs required treatment in lieu of dosage and/or schedule modification. To estimate the cost of each TEAE, the number of each treatment resource was multiplied by the cost per resource and summed across all resources used for treatment. ${ }^{40}$ All costs were inflated to 2018 values using the PCE inflation index. ${ }^{37}$ Unless otherwise noted, $100 \%$ of patients with a TEAE were assumed to have used each resource. Severe TEAEs included in the model, the percentage of patients experiencing each TEAE, and the cost to treat an instance of each TEAE are shown in Supplementary Table 1. Additional details describing the resources used to treat and follow-up on each TEAE and the unit cost for each resource used are shown in Supplementary Table 2.

\section{Results \\ Population}

In the base-case analysis for a private payer plan with one million members, the annual number of patients considered eligible for selinexor was four. ${ }^{14}$ In the base-case analysis from the Medicare perspective (51 million members), the annual number of patients considered eligible for selinexor was 159 .

\section{Costs}

In the base-case analysis, the total drug cost over the course of selinexor treatment was $\$ 44,079.30$ per patient. Treatment of severe TEAEs (ie, grade 3 or higher occurring in $\geq 5 \%$ of selinexor-treated patients) was estimated to cost $\$ 2,746.36$ per patient over the course of treatment. The

Table I Default Inputs for Treatment Dose, Duration, and Calculated Average Drug Costs per Patient

\begin{tabular}{|l|l|l|l|l|}
\hline Drug & Weekly Dose $^{\mathrm{a}}$ & $\begin{array}{l}\text { Treatment } \\
\text { Duration }^{\mathbf{a}}\end{array}$ & $\begin{array}{l}\text { Average Drug Cost per } \\
\text { Week }\end{array}$ & $\begin{array}{l}\text { Average Drug Cost per 8-Week } \\
\text { Treatment }^{\text {d }}\end{array}$ \\
\hline Selinexor & $\begin{array}{l}8 \text { Tablets of } 20 \mathrm{mg} \\
\text { strength } \\
(160 \mathrm{mg} \text { total })\end{array}$ & 8 weeks & $\$ 5,500^{\mathrm{b}}$ & $\$ 44,000.00$ \\
\hline Dexamethasone & $\begin{array}{l}10 \text { Tablets of } 4 \mathrm{mg} \\
\text { strength } \\
(40 \mathrm{mg} \text { total })\end{array}$ & 8 weeks & $\$ 9.91^{\mathrm{c}}$ & $\$ 79.30$ \\
\hline
\end{tabular}

Notes: ${ }^{a}$ Treatment dose and duration are based on the STORM clinical trial (NCT023368/5). ${ }^{33}$ Adherence of $100 \%$ is an assumption of the model. ${ }^{b}$ Intermediate calculation based on selinexor package size of 32 tablets of $20 \mathrm{mg}$ dose, with a package cost of $\$ 22,000 .^{35}$ Intermediate calculation based on dexamethasone package size of 100 tablets per package, with a package cost of $\$ 99.12 .{ }^{34}$ Calculated costs are based on the respective average drug costs per week and treatment duration. 
estimated annual cost of best supportive care was $\$ 6,893$ per patient. ${ }^{26}$ Thus, the total annual cost of selinexor treatment per patient (ie, the sum of drug costs, TEAE treatment costs, along with continuous best supportive care costs) is calculated to be $\$ 53,718.73$. The estimated PPPM cost with access to selinexor was $\$ 4,476.56$. The predominant contributor, $82 \%$ of the estimated PPPM cost, was drug costs.

\section{Net Budget Impact}

For a hypothetical US private payer with one million members, the total plan budget impact and PMPM impact are presented in Table 2. Over 3 years, the total cost of selinexor uptake was estimated at $\$ 347,111$, whereas the total cost of best supportive care was $\$ 44,541$. This resulted in a net budget impact of selinexor use of $\$ 302,571$ over the threeyear time horizon. The estimated PMPM impact was $\$ 0.0045$ for year $1, \$ 0.0103$ for year 2 , and $\$ 0.0103$ for year 3 .

In the base-case analysis for Medicare with 51 million members (Medicare Part D for 2018), the total budget impact and PMPM impact are presented in Table 3. Over 3 years, the total cost of selinexor uptake was estimated at
$\$ 13.35$ million, whereas the total cost of best supportive care was $\$ 1.71$ million. This resulted in a net budget impact of selinexor use of $\$ 11.64$ million over the three-year time horizon. The estimated PMPM impact was $\$ 0.0034$ for year $1, \$ 0.0078$ for year 2 , and $\$ 0.0078$ for year 3 .

\section{Scenario Analysis}

The model population was modified to reflect the number of patients eligible for selinexor. The first scenario expanded eligibility to include patients with triple-class refractory MM who have received three previous lines of therapy. A total of 10 patients were eligible for selinexor treatment in a private payer plan with one million members. The net budget impact of selinexor over 3 years was $\$ 756,426.92$. The PMPM was $\$ 0.0113$ for year $1, \$ 0.0259$ for year 2, and $\$ 0.0259$ for year 3. From the Medicare perspective with 51 million members, a total of 398 patients were considered eligible for treatment with selinexor. The net budget impact over 3 years was $\$ 29.10$ million. The PMPM was $\$ 0.0085$ for year 1 , $\$ 0.0195$ for year 2 , and $\$ 0.0195$ for year 3 .

Table 2 Base-Case Total Population Budget Impact and PMPM Impact for a Private Payer

\begin{tabular}{|l|l|l|l|l|l|l|l|}
\hline & \multicolumn{2}{l}{ Year I } & \multicolumn{2}{l|}{ Year 2 } & \multicolumn{2}{l|}{ Year 3 } & Total Years I-3 \\
\cline { 2 - 8 } & Total Cost & PMPM & Total Cost & PMPM & Total Cost & PMPM & Total Cost \\
\hline With access to selinexor & $\$ 62,302.04$ & $\$ 0.0052$ & $\$ 142,404.66$ & $\$ 0.0119$ & $\$ 142,404.66$ & $\$ 0.0119$ & $\$ 347,111.36$ \\
Drug costs & $\$ 51,122.39$ & $\$ 0.0043$ & $\$ 116,851.18$ & $\$ 0.0097$ & $\$ 116,851.18$ & $\$ 0.0097$ & $\$ 284,824.76$ \\
AE costs & $\$ 3185.18$ & $\$ 0.0003$ & $\$ 7280.41$ & $\$ 0.0006$ & $\$ 7280.41$ & $\$ 0.0006$ & $\$ 17,746.01$ \\
Best supportive care costs & $\$ 7994.47$ & $\$ 0.0007$ & $\$ 18,273.06$ & $\$ 0.0015$ & $\$ 18,273.06$ & $\$ 0.0015$ & $\$ 44,540.59$ \\
\hline Without access to selinexor & $\$ 7,994.47$ & $\$ 0.0007$ & $\$ 18,273.06$ & $\$ 0.0015$ & $\$ 18,273.06$ & $\$ 0.0015$ & $\$ 44,540.59$ \\
Best supportive care costs & $\$ 7,994.47$ & $\$ 0.0007$ & $\$ 18,273.06$ & $\$ 0.0015$ & $\$ 18,273.06$ & $\$ 0.0015$ & $\$ 44,540.59$ \\
\hline Budget impact & $\$ 54,307.57$ & $\$ 0.0045$ & $\$ 124,131.60$ & $\$ 0.0103$ & $\$ 124,131.60$ & $\$ 0.0103$ & $\$ 302,570.77$ \\
\hline
\end{tabular}

Note: The private payer perspective assumes a plan size of one million members.

Table 3 Base-Case Total Population Budget Impact and PMPM Impact for Medicare Part D

\begin{tabular}{|l|l|l|l|l|l|l|l|}
\hline & \multicolumn{2}{|l|}{ Year I } & \multicolumn{2}{l|}{ Year 2 } & \multicolumn{2}{l|}{ Year 3 } & Total Years I-3 \\
\cline { 2 - 8 } & Total Cost & PMPM & Total Cost & PMPM & Total Cost & PMPM & Total Cost \\
\hline With access to selinexor & $\$ 2,396,988.97$ & $\$ 0.0039$ & $\$ 5,478,831.93$ & $\$ 0.0090$ & $\$ 5,478,831.93$ & $\$ 0.0090$ & $\$ 13,354,652.83$ \\
Drug costs & $\$ 1,966,866.82$ & $\$ 0.0032$ & $\$ 4,495,695.58$ & $\$ 0.0073$ & $\$ 4,495,695.58$ & $\$ 0.0073$ & $\$ 10,958,257.98$ \\
AE costs & $\$ 122,545.62$ & $\$ 0.0002$ & $\$ 280,104.28$ & $\$ 0.0005$ & $\$ 280,104.28$ & $\$ 0.0005$ & $\$ 682,754.19$ \\
Best supportive care costs & $\$ 307,576.53$ & $\$ 0.0005$ & $\$ 703,032.06$ & $\$ 0.001 I$ & $\$ 703,032.06$ & $\$ 0.0011$ & $\$ 1,713,640.66$ \\
\hline Without access to selinexor & $\$ 307,576.53$ & $\$ 0.0005$ & $\$ 703,032.06$ & $\$ 0.0011$ & $\$ 703,032.06$ & $\$ 0.0011$ & $\$ 1,713,640.66$ \\
Best supportive care costs & $\$ 307,576.53$ & $\$ 0.0005$ & $\$ 703,032.06$ & $\$ 0.001 I$ & $\$ 703,032.06$ & $\$ 0.0011$ & $\$ 1,713,640.66$ \\
\hline Budget impact & $\$ 2,089,412.44$ & $\$ 0.0034$ & $\$ 4,775,799.87$ & $\$ 0.0078$ & $\$ 4,775,799.87$ & $\$ 0.0078$ & $\$ 11,641,012.17$ \\
\hline
\end{tabular}

Note: The Medicare Part D perspective assumes a plan size of 51 million members. 
In the second scenario, eligibility was extended to patients with triple-class refractory MM regardless of the number of prior lines of therapy. A total of 16 patients were eligible for selinexor treatment in a private payer plan with one million members. The net budget impact of selinexor over 3 years was $\$ 1,134,640.38$. The PMPM was $\$ 0.0170$ for year $1, \$ 0.0388$ for year 2 , and $\$ 0.0388$ for year 3. From the Medicare perspective with 51 million members, a total of 598 patients were considered eligible for selinexor treatment. The net budget impact over 3 years was $\$ 43.65$ million. The PMPM was $\$ 0.0128$ for year 1, $\$ 0.0293$ for year 2, and $\$ 0.0293$ for year 3 .

\section{One-Way Sensitivity Analysis}

The duration of treatment, uptake of selinexor in year 1 after market entry, and per package cost of selinexor were varied by $\pm 10 \%$ from baseline to gauge the impact on total costs for a private payer with a plan population of one million members. The base-case total population budget impact in the first year after selinexor introduction was found to be $\$ 54,307.57$ (Table 2). The results of the oneway sensitivity analysis are summarized in Figure 2 and Supplementary Table 3 . The year 1 budget impact had comparable sensitivity to the variations modeled in the three input parameters. The maximum budget impact, $\$ 59,738.33$, was observed when uptake in year 1 was increased by $10 \%$ from the base-case input (ie, from $28 \%$ to $30.8 \%$ ).

\section{Discussion}

Understanding the potential budget impact of new therapies is important from the perspective of healthcare decision-makers in managing spending and assessing the value of treatments. The budget impact model herein examined the anticipated costs of adopting selinexor into a US payer plan for the treatment of patients with penta-refractory MM. For a hypothetical private payer with a total plan population of one million members, the budget impact associated with the introduction of selinexor was estimated to be $\$ 302,571$ over a threeyear period. This was associated with a PMPM cost of $\$ 0.0103$ for year 3. In the base-case analysis for Medicare with a total population of 51 million members, the budget impact was estimated to be $\$ 11.64$ million over a threeyear period, with a PMPM cost of $\$ 0.0078$ for year 3 . The estimated PPPM cost of selinexor in the current analysis was $\$ 4,476.56$.

Other pharmacoeconomic studies have estimated the budget impact of various therapy options for patients with RRMM. Shao et al (2016) identified PPPM costs ranging from $\$ 13,377$ to $\$ 25,850$ for lenalidomide-, bortezomib-, carfilzomib-, and pomalidomide-based regimens in patients with RRMM in the third-line of therapy. ${ }^{41}$ Bloudek et al (2016) estimated a year 1 PMPM incremental cost of $<\$ 0.01$ for the introduction of panobinostat into a US third-party payer plan. The budget impact model examined direct costs

TOTAL POPULATION BUDGET IMPACT IN USD
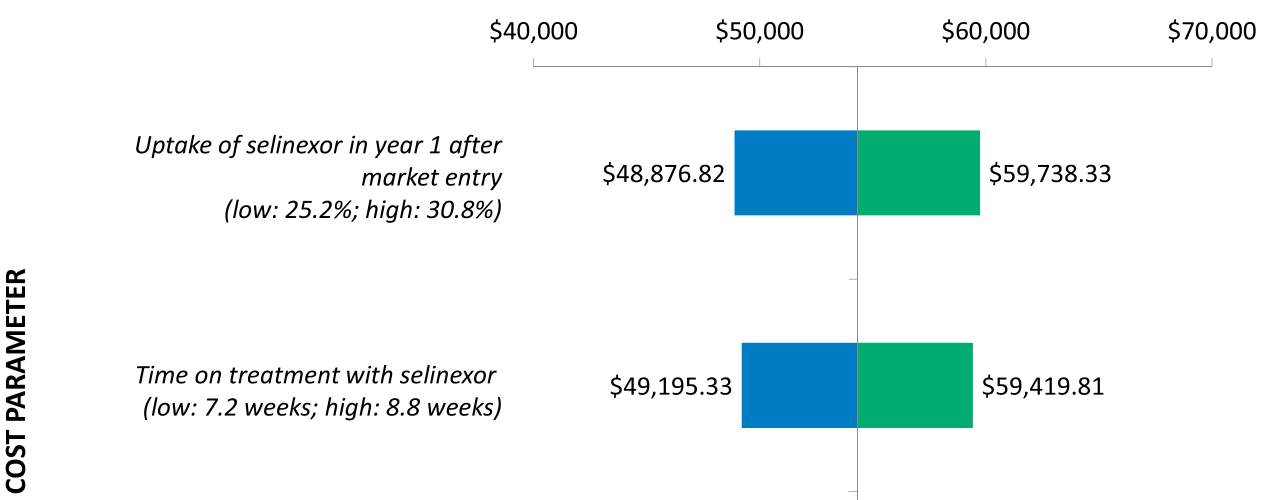

Cost of selinexor package (low: $\$ 19,800.00$; high: $\$ 24,200.00)$

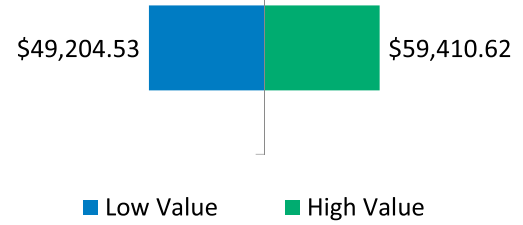

Figure 2 One-way sensitivity analysis results of total population budget impact in year I after selinexor introduction for a private payer. 
of treatment (ie, drug costs, TEAE management and treatment) for a hypothetical US payer with one million members, with an annual number of 16 patients eligible for treatment. $^{42}$ Potluri et al (2016) estimated an PMPM budget impact of $\$ 0.032$ in year 1 for the introduction of elotuzumab in combination with lenalidomide and dexamethasone into a hypothetical payer plan with one million members and 29 patients eligible for treatment (9.7\% uptake). ${ }^{43}$ Hollmann et al (2017) examined the budget impact of adding daratumumab to combination therapy regimens from the perspective of a US payer over a 1-year time horizon. ${ }^{44}$ For a hypothetical payer with one million members and 141 eligible patients, the estimated PMPM cost was $\$ 0.08$, corresponding to the sum of drug costs, administration costs, and TEAE management.

Selinexor is currently the only approved therapy for adult patients with penta-refractory MM in the US. As a result, it is not possible to make a direct comparison of the budget impact of selinexor with that of other therapies for this indication. It is important to consider the rapidly evolving treatment landscape for RRMM and the development of new modalities such as chimeric antigen T-cell (CAR-T) therapy or targeted therapies against B-cell antigen maturation factor (BCMA). ${ }^{45}$ The budget impact of selinexor and its market share are expected to shift over time with the approval and uptake of newer therapies for the treatment of pentarefractory RRMM. It is notable that in the current analysis, the budget impact of selinexor remained modest in a scenario analysis in which the indication of selinexor was extended to patients with triple-class refractory MM regardless of the number of prior lines of therapy. In the case of a private payer plan with one million members and 16 eligible patients, the estimated year 3 PMPM cost was $\$ 0.0388$. From the Medicare perspective with 51 million members and 598 eligible patients, the estimated year 3 PMPM cost was \$0.0293. Collectively, these findings suggest a modest budget impact resulting from the introduction of selinexor into a US third-party payer plan.

\section{Limitations}

This model examined direct costs associated with selinexor use (ie, drug price and TEAE management), as well as variations in market uptake, drug price, and treatment duration. There were no assumed cost offsets associated with selinexor treatment or a reduction in the cost of best supportive care. The model did not consider factors such as clinical response, mortality, and secondary clinical endpoints. The costs associated with an increase in patient life span were also not included. As no reasonable alternative treatment exists for patients with penta-refractory MM, costs of selinexor treatment could only be compared to best supportive treatment costs alone.

\section{Conclusion}

This analysis demonstrates the small and manageable budget impact of selinexor for a third-party US payer, given the low prevalence of penta-refractory MM. For a relatively low PMPM impact, selinexor provides clinical benefit for some patients with penta-refractory MM and fulfills a previously unmet therapeutic need for this patient population.

\section{Data Sharing Statement}

The data presented in this publication are publicly available.

\section{Ethical Declaration}

Review and approval by an institutional review board or ethics committee were not required for this research. The research does not involve patients/people, medical records, or human samples.

\section{Acknowledgments}

Nesrin Vurgun of Purple Squirrel Economics provided editorial support. Jatin Shah of Karyopharm Therapeutics Inc. provided a critical review of the manuscript.

\section{Funding}

This study was funded by Karyopharm Therapeutics.

\section{Disclosure}

Parts of this study were previously presented at the American Society of Hematology conference and later published in abstract form in an issue supplement of Blood (Volume 134, November 2019, page 5824). J. Mehta and J. Bassali are employees of Karyopharm Therapeutics. I. Gould, J.A. Kaye, and D. Mladsi are employees of RTI International, which received funding from Karyopharm Therapeutics to conduct this study. The authors report no other possible conflicts of interest in this work. 


\section{References}

1. Kumar SK, Rajkumar V, Kyle RA, et al. Multiple myeloma. Nat Rev Dis Primer. 2017;3(1):17046. doi:10.1038/nrdp.2017.46

2. Surveillance, Epidemiology, and End Results (SEER). Program. Cancer stat facts: myeloma. 2016. Available from: https://seer.can cer.gov/statfacts/html/mulmy.html. Accessed November 27, 2018.

3. Cancer facts \& Figures 2019. Available from: http://www.cancer.org/ content/dam/cancer-org/research/cancer-facts-and-statistics/annualcancer-facts-and-figures/2019/cancer-facts-and-figures-2019.pdf. Accessed October 30, 2019.

4. Dimopoulos MA, Richardson PG, Moreau P, Anderson KC. Current treatment landscape for relapsed and/or refractory multiple myeloma. Nat Rev Clin Oncol. 2015;12(1):42-54. doi:10.1038/nrclinonc.2014.200

5. Sonneveld P, De Wit E, Moreau P. How have evolutions in strategies for the treatment of relapsed/refractory multiple myeloma translated into improved outcomes for patients? Crit Rev Oncol Hematol. 2017;112:153-170. doi:10.1016/j.critrevonc.2017.02.007

6. Fonseca R, Abouzaid S, Bonafede M, et al. Trends in overall survival and costs of multiple myeloma, 2000-2014. Leukemia. 2017;31 (9):1915-1921. doi:10.1038/leu.2016.380

7. Mikhael J. Treatment options for triple-class refractory multiple myeloma. Clin Lymphoma Myeloma Leuk. 2019;\$215226501932 0087. doi:10.1016/j.clml.2019.09.621

8. Bazarbachi AH, Al Hamed R, Malard F, Harousseau J-L, Mohty M. Relapsed refractory multiple myeloma: a comprehensive overview. Leukemia. 2019;33(10):2343-2357. doi:10.1038/s41375-019-0561-2

9. Kumar SK, Dimopoulos MA, Kastritis E, et al. Natural history of relapsed myeloma, refractory to immunomodulatory drugs and proteasome inhibitors: a multicenter IMWG study. Leukemia. 2017;31 (11):2443-2448. doi:10.1038/leu.2017.138

10. Cornell RF, Gandhi UH, Lakshman A, et al. Subsequent treatment outcomes of multiple myeloma refractory to CD38-monoclonal antibody therapy. Blood. 2018;132(Supplement 1):2015. doi:10.1182/ blood-2018-99-115078

11. Gandhi UH, Cornell RF, Lakshman A, et al. Outcomes of patients with multiple myeloma refractory to CD38-targeted monoclonal antibody therapy. Leukemia. 2019;33(9):2266-2275. doi:10.1038/s41375-0190435-7

12. Pick M, Vainstein V, Goldschmidt N, et al. Daratumumab resistance is frequent in advanced-stage multiple myeloma patients irrespective of CD38 expression and is related to dismal prognosis. Eur J Haematol. 2018;100(5):494-501. doi:10.1111/ejh.13046

13. Usmani S, Ahmadi T, Ng Y, et al. Analysis of real-world data on overall survival in multiple myeloma patients with $\geq 3$ prior lines of therapy Including a Proteasome Inhibitor (PI) and an Immunomodulatory Drug (IMID), or double refractory to a PI and an imid. The Oncologist. 2016;21(11):1355-1361. Doi:10.1634/ theoncologist.2016-0104

14. XPOVIO (selinexor) prescribing information. https://www.access data.fda.gov/drugsatfda_docs/label/2019/212306s000lbl.pdf. Available from: Accessed July. 8, 2019.

15. Chari A, Vogl DT, Gavriatopoulou M, et al. Oral selinexor-dexamethasone for triple-class refractory multiple myeloma. $N$ Engl J Med. 2019;381(8):727-738. doi:10.1056/NEJMoa1903455

16. Jagannath S, Vogl DT, Dimopoulos M-A, et al. Phase $2 \mathrm{~b}$ results of the STORM study: oral selinexor plus low dose dexamethasone (SD) in patients with penta-refractory myeloma (Penta-MM). Clin Lymphoma Myeloma Leuk. 2018;18:S249-S250. doi:10.1016/j.clml.2018.07.149

17. Runyan A, Banks J, Bruni DS. Current and future oncology management in the United States. J Manag Care Spec Pharm. 2019;25 (2):272-281. doi:10.18553/jmcp.2019.25.2.272

18. Djatche LM, Goble JA, Chun G, Varga S. Evaluating oncology value-based frameworks in the U.S. marketplace and challenges in real-world application: a multiple myeloma test case. J Manag Care Spec Pharm. 2018;24(1):39-46. doi:10.18553/jmcp.2018.24.1.39
19. Schnipper LE, Davidson NE, Wollins DS, et al. American Society of Clinical Oncology Statement: a conceptual framework to assess the value of cancer treatment options. J Clin Oncol. 2015;33 (23):2563-2577. doi:10.1200/JCO.2015.61.6706

20. Schnipper LE, Davidson NE, Wollins DS, et al. Updating the American Society of Clinical Oncology value framework: revisions and reflections in response to comments received. J Clin Oncol. 2016;34(24):2925-2934. doi:10.1200/JCO.2016.68.2518

21. Sullivan SD, Mauskopf JA, Augustovski F, et al. Budget impact analysis-principles of good practice: report of the ISPOR 2012 budget impact analysis good Practice II task force. Value Health. 2014;17(1):5-14. doi:10.1016/j.jval.2013.08.2291

22. Mauskopf JA, Sullivan SD, Annemans L, et al. Principles of good practice for budget impact analysis: report of the ISPOR task force on good research practices-budget impact analysis. Value Health. 2007;10(5):336-347. doi:10.1111/j.1524-4733.2007.00187.x

23. The AMCP format for formulary submissions, version 3.1. A format for submission of clinical and economic evidence of pharmaceuticals in support of formulary consideration. December 2012. Available from: http://amcp.org/practice-resources/amcp-format-formularysubmisions.pdf. Accessed July 16, 2018.

24. MacEwan JP, Batt K, Yin W, et al. Economic burden of multiple myeloma among patients in successive lines of therapy in the United States. Leuk Lymphoma. 2018;59(4):941-949. doi:10.1080/1042819 4.2017.1361035

25. 2017 National Population Projections Datasets. Available from: https://www.census.gov/data/datasets/2017/demo/popproj/2017popproj.html. Accessed January 29, 2019.

26. Karyopharm Therapeutics, Inc. Epidemiology presentation. Data on file. 2018a.

27. Karyopharm Therapeutics, Inc. Updated MM Epidemiology Presentation based on Symphony claims analysis (2016-2018); FHAD (2011-2018); STORM recruitment; and market research. Data on file; 2019.

28. Cid Ruzafa J, Merinopoulou E, Baggaley RF, et al. Patient population with multiple myeloma and transitions across different lines of therapy in the USA: an epidemiologic model: multiple Myeloma lines of therapy. Pharmacoepidemiol Drug Saf. 2016;25(8):871-879. doi:10. 1002/pds.3927

29. Costa LJ, Brill IK, Omel J, Godby K, Kumar SK, Brown EE. Recent trends in multiple myeloma incidence and survival by age, race, and ethnicity in the United States. Blood Adv. 2017;1(4):282-287. doi:10.1182/bloodadvances.2016002493

30. Kazandjian D. Multiple myeloma epidemiology and survival: A unique malignancy. Semin Oncol. 2016;43(6):676-681. doi:10.10 53/j.seminoncol.2016.11.004

31. Cubanski J, Damico A, Neuman T. Medicare Part D: A first look at prescription drug plans in 2019. Available from: https://www.kff.org/ medicare/issue-brief/medicare-part-d-a-first-look-at-prescription-drug -plans-in-2019/. Accessed February 1, 2019.

32. Center for Medicare and Medicaid Services, Fast Facts. January 2019. Available from: https://www.cms.gov/Research-Statistics-Dataand-Systems/Statistics-Trends-and-Reports/CMS-Fast-Facts/index. html. Accessed May 18, 2020. 7, 2019.

33. Selinexor Treatment of Refractory Myeloma (STORM). August 2018. Available from: https://clinicaltrials.gov/ct2/show/ NCT02336815?term=NCT02336815\&rank=1. Accessed November 21, 2018.

34. Micromedex 2.0. Red Book Online. Thomson Reuters. Available from: https://www.micromedexsolutions.com. Accessed December $17,2018$.

35. Karyopharm Therapeutics, Inc. Email correspondence regarding pricing. Data on file. 2018c.

36. Mauskopf J, Earnshaw SR, Brogan AJ, Wolowacz S, Brodtkorb T-H Budget-Impact Analysis of Health Care Interventions: A Practical Guide; 2017. 
37. Bureau of Economic Analysis, 2018: Table 2.4.4U. Price Indexes for Personal Consumption Expenditures by Type of Product. December 2018.

38. Karyopharm Therapeutics, Inc. US Demand Estimation Presentation. Data on file, 2018d.

39. Karyopharm Therapeutics, Inc. KCP-330-012 CSR. Data on file. 2018 b.

40. Patterns of Health Care Resource Use Required for Treatment of Each TEAE Based on Guidance from James A. Kaye, MD (Hematology, Oncology). DrPH, RTI Health Solutions.

41. Shao C, Monberg M, Cao X, Zhou W, Zhong Y, Marinello P. Realworld treatment patterns, health care utilization, and costs among relapsed/Refractory Multiple Myeloma (rrMM) patients. Blood. 2016;128(22):3555. doi:10.1182/blood.V128.22.3555.3555

42. Bloudek L, Roy A, Kish JK, et al. Estimating the economic impact of adding panobinostat to a U.S. formulary for relapsed and/or refractory multiple myeloma: a budget impact and cost-benefit model. J Manag Care Spec Pharm. 2016;22(8):991-1002. doi:10.18553/ jmcp.2016.22.8.991
43. Potluri R, Bhandari H, Ranjan S, Chen C. Budget impact analysis of introducing elotuzumab in combination with lenalidomide and dexamethasone for relapsed/refractory multiple myeloma: a US payer perspective. Blood. 2016;128(22):2363. doi:10.1182/blood.V128.22. 2363.2363

44. Hollmann S, Goyert N, Moldaver D, Grima D, Maiese E. U.S. budget impact analysis for daratumumab in combination with lenalidomide and dexamethasone, bortezomib and dexamethasone, or pomalidomide and dexamethasone for the treatment of patients with relapsed/ refractory multiple myeloma. In: meeting Abstracts - Academy of Managed Care Pharmacy Nexus 2017. Vol 23. Journal of Managed Care \& Specialty Pharmacy. 2017;S32. doi:10.18553/jmcp.2017.23. 10 -a.s1

45. Shah N, Chari A, Scott E, Mezzi K, Usmani SZ. B-cell maturation antigen (BCMA) in multiple myeloma: rationale for targeting and current therapeutic approaches. Leukemia. 2020;34(4):985-1005. doi:10.1038/s41375-020-0734-Z
ClinicoEconomics and Outcomes Research

\section{Publish your work in this journal}

ClinicoEconomics and Outcomes Research is an international, peerreviewed open-access journal focusing on Health Technology Assessment, Pharmacoeconomics and Outcomes Research in the areas of diagnosis, medical devices, and clinical, surgical and pharmacological intervention. The economic impact of health policy and health systems
Dovepress

organization also constitute important areas of coverage. The manuscript management system is completely online and includes a very quick and fair peer-review system, which is all easy to use. Visit $\mathrm{http}: / / \mathrm{www}$.dovepress.com/testimonials.php to read real quotes from published authors. 